\title{
BRST quantization and equivariant cohomology: localization with asymptotic boundaries
}

\author{
Bernard de Wit, ${ }^{a, b}$ Sameer Murthy ${ }^{c}$ and Valentin Reys ${ }^{d, e}$ \\ ${ }^{a}$ Nikhef Theory Group, \\ Science Park 105, 1098 XG Amsterdam, The Netherlands \\ ${ }^{b}$ Institute for Theoretical Physics, Utrecht University, \\ Leuvenlaan 4, 3584 CE Utrecht, The Netherlands \\ ${ }^{c}$ Department of Mathematics, King's College London, \\ The Strand, London WC2R 2LS, U.K. \\ ${ }^{d}$ Dipartimento di Fisica, Università di Milano-Bicocca, \\ Piazza della Scienza 3, I-20126 Milano, Italy \\ ${ }^{e}$ INFN - Sezione di Milano-Bicocca, \\ Piazza della Scienza 3, I-20126 Milano, Italy. \\ E-mail: b.dewit@uu.nl, sameer.murthy@kcl.ac.uk, \\ valentin.reys@unimib.it
}

ABSTRACT: We develop BRST quantization of gauge theories with a soft gauge algebra on spaces with asymptotic boundaries. The asymptotic boundary conditions are imposed on background fields, while quantum fluctuations about these fields are described in terms of quantum fields that vanish at the boundary. This leads us to construct a suitable background field formalism that is generally applicable to soft gauge algebras, and therefore to supergravity. We define a nilpotent BRST charge that acts on both the background and the quantum fields, as well as on the background and quantum ghosts.

When the background is restricted to be invariant under a residual isometry group, the background ghosts must be restricted accordingly and play the role of the parameters of the background isometries. Requiring in addition that the background ghosts will be BRST invariant as well then converts the BRST algebra into an equivariant one. The background fields and ghosts are then invariant under the equivariant transformations while the quantum fields and ghosts transform under both the equivariant and the background transformations. We demonstrate how this formalism is suitable for carrying out localization calculations in a large class of theories, including supergravity defined on asymptotic backgrounds that admit supersymmetry.

KEYwords: BRST Quantization, Gauge Symmetry, Supergravity Models, Supersymmetric Gauge Theory

ARXIV EPRINT: 1806.03690 


\section{Contents}

1 Introduction 1

2 BRST cohomology for soft algebras 3

$\begin{array}{llr}3 & \text { The background field split } & 6\end{array}$

4 Equivariant cohomology 10

5 Localization of the functional integral $\quad 14$

6 Application to exact quantum entropy of supersymmetric black holes 18

\section{Introduction}

The standard quantization of gauge theories, especially in the context of perturbation theory, is carried out by imposing suitable gauge conditions that require the introduction of so-called ghost fields [1-3]. The theory is then no longer invariant under local gauge transformations, but under a rigid fermionic nilpotent variation $\delta_{\text {brst }}$ known as BRST symmetry $[4,5]$. When the generators of the gauge group close under commutation, the quantum action involves terms that are bilinears of ghost and anti-ghost fields. The BRST variations of the original fields can be directly expressed in terms of the original gauge transformations with their parameters replaced by the ghost fields. The partition function for BRST invariant operators as well as the S-matrix are then independent of the gauge condition. ${ }^{1}$

The formal structure of BRST transformations can in certain cases also be used in the study of topological theories, where one has a nilpotent fermionic operator $\delta$, often arising as a twisted supercharge of some supersymmetric theory $[11,12]$. Here the ghosts will usually not originate from quantizing the theory, but they are provided by the matter fermions of the original theory. The functional integral then localizes to the $\delta$-cohomology. More generally, one can consider a fermionic symmetry operator $\delta_{\text {eq }}$ with algebra $\delta_{\text {eq }}{ }^{2}=\delta_{\dot{\xi}}$, where $\delta_{\xi}$ is the generator of a compact bosonic symmetry. In this case one can apply the powerful mathematical framework of equivariant localization [13-15], with the result that the functional integral will localize to the $\delta_{\text {eq }}$-cohomology. This technique has been used to great effect in the context of supersymmetric gauge theories [16], by choosing $\delta_{\xi}$ to be a combination of a compact isometry and internal symmetry variations. These techniques can be extended to supersymmetric theories on curved manifolds admitting non-trivial rigid symmetries [17].

\footnotetext{
${ }^{1}$ When the gauge algebra closes only modulo the equations of motion, then additional terms will be required of higher order in the ghost and anti-ghost fields in both the action and the BRST transformations. In that case one is dealing with an open BRST algebra [6-9]. For a review, see [10].
} 
These developments have led to a large number of applications, but essentially all of them deal with rigid supersymmetry (see the review [18] for a collection of recent results). In this paper we lay out a formalism for local supersymmetry, which can account for the fluctuations of (super-)gravitons in the path integral. We were motivated to study this problem in the context of applying localization to determine the exact entropy of BPS black holes [19] in supergravity - a program which has already produced interesting results $[20,21]$, but where the underlying formalism needs to be put on a more rigorous footing. Hence the focus in this paper will be on the complications that one encounters when attempting to apply localization to theories with fluctuating (super-)gravitons.

The power of the localization method is that it reduces an infinite-dimensional functional integral to an integral over $\delta_{\text {eq }}$-invariant field configurations. This is an enormous reduction which, in lucky situations, could even lead to a finite-dimensional integral. Field configurations that are $\delta_{\text {eq }}$-invariant are necessarily $\delta_{\xi}$-invariant, and an appropriate choice of the background bosonic symmetries $\delta_{\xi}$ constrains the field configurations to fluctuate only along a restricted set of directions in space-time as well as in field space. In theories of supergravity, however, the meaning of $\delta_{\text {eq }}$ and $\delta_{\xi}$ are not a priori clear, as both supersymmetry as well as space-time translations are part of the gauge algebra of supergravity. One situation in which we can make sense of a rigid symmetry in a gravitational theory is to consider a space with a boundary and fix the behavior of all the fields near the boundary. $^{2}$ The functional integral is then performed over all the field fluctuations about a fixed background field configuration that satisfies the boundary conditions.

A first natural step in this situation would be to recast the problem in the background field formalism. In trying to work out the details, however, we run into a technical hurdle, namely that a general understanding of the background field method is lacking for gauge theories with soft algebras, i.e. theories in which the structure 'constants' of the gauge algebra are functions of fields (as is the case for supergravity). We solve this problem by constructing a nilpotent BRST operator for soft gauge algebras in a situation where the fields have been split into background and quantum pieces, and by introducing two corresponding sets of ghosts. The BRST operator then acts on both the classical and the quantum fields, as well as on the two sets of ghosts. Subsequently we consider a functional integral that only depends on the background fields (but not, as it turns out, on the background ghosts), which is gauge independent provided the background fields are invariant. As a next step we deform the BRST operator to an equivariant symmetry $\delta_{\text {eq }}$, by appropriately freezing the background fields and ghosts, so as to obtain a rigid supersymmetry algebra of the boundary, with an action on the full space of classical as well as quantum fields. Our construction is very general in that it provides a framework for equivariant localization for any gauge algebra (including soft algebras) with some choice of a rigid subalgebra that is picked by the boundary.

At a technical level, our problem involves setting up the action of $\delta_{\text {eq }}$ on the set of all fields in the gauge-fixed theory, and computing the $\delta_{\text {eq }}$-cohomology. Different methods

\footnotetext{
${ }^{2}$ In the context of AdS/CFT this is particularly natural, and, as is well-known, the space of boundary configurations of the bulk gravitational theory couples to the non-gravitational theory and thus inherits its rigid symmetries.
} 
have been used in the past to solve this cohomology problem, including BRST-based methods [22]. We refer the reader to [23] for a comprehensive review. The main new points that we discuss in this paper are functional integrals in theories with soft gauge algebras, and the general mechanism of how background symmetries act on quantum fields. The application to localization in supergravity can then be accomplished by specializing to a subalgebra of the background isometries that contains a supersymmetry which squares to a compact background isometry. We then show that the functional integral localizes to the

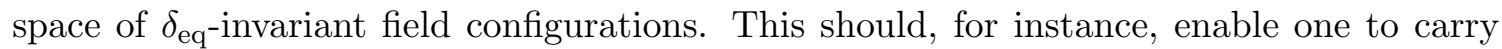
out a first-principles calculation of the exact quantum entropy of half-BPS black holes in $\mathcal{N}=2$ supergravity, thus completing the analysis of $[20,21]$. Although the present paper is inspired by thinking about localization for BPS black holes in supergravity, we should stress that we present a rather general formalism that can equally well be used in a broader context.

The plan of the paper is as follows. In section 2 we present a brief review of BRST quantization for soft gauge algebras and establish the notation. For simplicity we restrict ourselves to bosonic gauge invariances only, but at the end of the section we indicate how to deal with the more general case. Subsequently we introduce the background field formulation in section 3. We define a functional integral that only depends on the background fields and that is independent of the gauge condition when the background fields are invariant. In section 4 we discuss an equivariant cohomology that arises upon a specific deformation where all the background fields and ghosts are invariant and the quantum fields and ghosts transform under $\delta_{\text {eq }}$, which squares to a background isometry $\delta_{\xi}$. Under certain conditions the functional integral introduced in section 3 is also invariant under this equivariant algebra. In the next section 5, we demonstrate how this equivariant algebra can be used for localization. Finally in section 6 we present further details on how to apply our method when determining BPS black hole entropy.

\section{BRST cohomology for soft algebras}

To introduce our notation we first define the BRST transformations in the generic case of a gauge theory of bosonic gauge transformations with a gauge algebra that closes off shell (i.e. without the need of imposing the field equations). Hence we have gauge transformations expressed in terms of corresponding space-time dependent parameters $\xi^{\alpha}(x)$. The infinitesimal gauge transformations of the fields $\phi^{i}$ are written as follows,

$$
\delta \phi^{i}=R(\phi)^{i} \xi^{\alpha},
$$

where $R(\phi)_{\alpha}^{i}$ may include derivatives acting on the parameters $\xi^{\alpha}(x)$ and may depend non-linearly on the fields $\phi^{i}$. They must satisfy the general closure relation

$$
\delta\left(\xi_{1}\right) \delta\left(\xi_{2}\right)-\delta\left(\xi_{2}\right) \delta\left(\xi_{1}\right)=\delta\left(\xi_{3}\right),
$$

with $\xi_{3}{ }^{\alpha}=f_{\beta \gamma}{ }^{\alpha} \xi_{1}{ }^{\beta} \xi_{2}{ }^{\gamma}$. The structure 'constants' $f_{\beta \gamma}{ }^{\alpha}$ may depend on $\phi^{i}$ and follow directly from the closure relation (2.2). This leads to the following result,

$$
R_{[\alpha}^{j} \partial_{j} R_{\beta]}^{i}=\frac{1}{2} f_{\alpha \beta}^{\gamma} R_{\gamma}^{i} .
$$


Upon applying a third infinitesimal gauge transformation one derives the corresponding Jacobi identity,

$$
f_{[\alpha \beta}^{\delta} f_{\gamma] \delta}^{\epsilon}+R_{[\alpha}^{j} \partial_{j} f_{\beta \gamma]}^{\epsilon}=0 .
$$

Gauge algebras with field-dependent structure constants are often called soft algebras. Supergravity theories are usually based on a soft gauge algebra. The closure relation (2.3) and the corresponding Jacobi identy (2.4) will play an important role throughout this paper.

The BRST transformations for the fields $\phi^{i}$ and the ghosts $c^{\alpha}$ then take the following form,

$$
\begin{aligned}
\delta_{\mathrm{brst}} \phi^{i} & =R(\phi)^{i}{ }_{\alpha} \Lambda c^{\alpha}, \\
\delta_{\mathrm{brst}} c^{\alpha} & =\frac{1}{2} f_{\beta \gamma}{ }^{\alpha} c^{\beta} \Lambda c^{\gamma} .
\end{aligned}
$$

Here we have introduced an auxiliary anti-commuting number $\Lambda$, so that the fields and their variations have the same statistics. Its presence also helps to keep track of the various minus signs that one will encounter in the calculations. It is straightforward to verify that the above transformations are nilpotent when acting on $\phi^{i}$ or $c^{\alpha}$ by virtue of (2.3) and (2.4),

$$
\delta_{\text {brst }}^{2} \phi^{i}=0, \quad \delta_{\text {brst }}^{2} c^{\alpha}=0 .
$$

To see this one applies two consecutive BRST transformations with anti-commuting parameters $\Lambda_{1}$ and $\Lambda_{2}$.

The gauge-invariant classical Lagrangian $\mathcal{L}^{\text {class }}(\phi)$ is obviously BRST invariant, because the BRST transformations on the fields $\phi^{i}$ take the form of an infinitesimal gauge transformation with field-dependent parameters. We allow for an arbitrary Lagrangian of this type, which may be formulated in space-times of various signatures. In addition we must include an extra BRST invariant term denoted by $\mathcal{L}^{\text {g.f. }}$ to fix the gauge, which will also provide the ghost-dependent terms in the full quantum action. This requires the introducion of anti-ghost fields $b_{\alpha}$ and Lagrange multiplier fields $B_{\alpha}$, which will also transform under nilpotent BRST transformations that we will define momentarily. The invariance of the action $\mathcal{L}^{\text {g.f. }}$ is then achieved by writing it as a BRST transformation of the so-called gauge fermion,

$$
\mathcal{L}^{\text {g.f. }}=\partial_{\Lambda} \delta_{\text {brst }}\left[b_{\alpha} F(\phi)^{\alpha}\right] .
$$

When $\delta_{\text {brst }} b_{\alpha}$ is proportional to $B_{\alpha}$, then $B_{\alpha}$ will indeed act as a Lagrange multiplier for the gauge choice $F(\phi)^{\alpha}=0$. Note that we have extracted the auxiliary anti-commuting number $\Lambda$ by a left derivative $\partial_{\Lambda}$.

Choosing $\delta_{\text {brst }} b_{\alpha}=\Lambda B_{\alpha}$ and $\delta_{\text {brst }} B_{\alpha}=0$, one ensures that the BRST transformations on $b_{\alpha}$ and $B_{\alpha}$ are indeed nilpotent. Subsequently one obtains the following expression for $\mathcal{L}^{\text {g.f. }}$,

$$
\mathcal{L}^{\text {g.f. }}=B_{\alpha} F(\phi)^{\alpha}-b_{\alpha} R(\phi)^{j}{ }_{\beta} c^{\beta} \partial_{j} F(\phi)^{\alpha},
$$

where we also assumed that the $\phi^{i}$ are commuting fields. The last term is precisely the Faddeev-Popov ghost Lagrangian [3]. Hence the BRST Lagrangian equals

$$
\mathcal{L}_{\text {brst }}\left(\phi^{i}, c^{\alpha}, b_{\alpha}, B_{\alpha}\right)=\mathcal{L}^{\text {class }}\left(\phi^{i}\right)+\mathcal{L}^{\text {g.f. }}\left(\phi^{i}, c^{\alpha}, b_{\alpha}, B_{\alpha}\right),
$$


which is invariant under the combined BRST transformations

$$
\begin{aligned}
\delta_{\mathrm{brst}} \phi^{i} & =R(\phi)^{i}{ }_{\alpha} \Lambda c^{\alpha}, & \delta_{\mathrm{brst}} b_{\alpha} & =\Lambda B_{\alpha}, \\
\delta_{\mathrm{brst}} c^{\alpha} & =\frac{1}{2} f_{\beta \gamma}{ }^{\alpha} c^{\beta} \Lambda c^{\gamma}, & \delta_{\mathrm{brst}} B_{\alpha} & =0 .
\end{aligned}
$$

The action corresponding to the Lagrangian (2.9) can be used to define a corresponding path integral by integrating over the various fields. Here it is important that the integral measure is also invariant under the BRST transformations. The BRST cohomolgy is based on the fact that the BRST transformations are nilpotent on all the fields.

The quantities $F(\phi)^{\alpha}$ are known as the gauge-fixing terms and ensure that the gauge invariance is removed. In principle this implies that the number of degrees of freedom will change, because the gauge fields will now acquire an additional degree of freedom. However, at the same time we have included a Lagrange multiplier field $B_{\alpha}$ of the same statistics as the corresponding gauge field, as well as a ghost field $c^{\alpha}$ and an anti-ghost field $b_{\alpha}$ of opposite statistics. Hence the difference between the numbers of bosonic and the number of fermionic degrees of freedom remains unchanged.

There may be additional problems when the gauge-fixing terms fail to fix all the gauge degrees of freedom entirely. In that case the ghost system will have a secondary gauge invariance which must be fixed by repeating the same procedure and introducing a next generation of ghost fields. Such a phenomenon is known to occur, for instance, for antisymmetric tensor gauge fields $[24,25]$. An elegant way to deal with this situation has been described in [9]. Furthermore the expectation values of the gauge-fixing terms must remain zero at the quantum level, so that the BRST symmetry will not be realized in a spontaneously broken way [26].

What remains is to consider the extension to the case of a gauge algebra with both bosonic and fermionic generators. In principle this extension is standard (see e.g. [10]), and we briefly introduce the relevant notation. Let us first consider the matter fields $\phi^{i}$, which can refer to either commuting (bosonic) or anti-commuting (fermionic) fields. To each field we assign a statistical index $\epsilon_{i}$, equal to 0 when the field is bosonic and to 1 when the field is fermionic, so that $\phi^{i} \phi^{j}=(-)^{\epsilon_{i} \epsilon_{j}} \phi^{j} \phi^{i}$. Likewise we introduce similar indices $\epsilon_{\alpha}$ for the transformation parameters. Note that these indices are defined modulo 2 . These definitions now enable one to define statistical indices for all quantities involved. For instance we have

$$
\epsilon\left(R_{\alpha}^{i}\right)=\epsilon_{i}+\epsilon_{\alpha}, \quad \epsilon\left(f_{\alpha \beta}{ }^{\gamma}\right)=\epsilon_{\alpha}+\epsilon_{\beta}+\epsilon_{\gamma}, \quad f_{\alpha \beta}^{\gamma}=(-)^{\epsilon_{\alpha}+\epsilon_{\beta}} f_{\beta \alpha}^{\gamma} .
$$

In the context of BRST the indices of the additional fields and the parameter follow directly from the definitions above,

$$
\epsilon\left(c^{\alpha}\right)=\epsilon\left(b_{\alpha}\right)=\epsilon_{\alpha}+1, \quad \epsilon\left(B_{\alpha}\right)=\epsilon_{\alpha}, \quad \epsilon(\Lambda)=1 .
$$

Finally we should point out that the derivative with respect to an anti-commuting quantity is ambiguous when acting on a commuting composite. In that case one has to distinguish between a right- and a left-derivative (whose sum will be vanishing).

Note that in the main body of the paper we assume that all the gauge field generators are bosonic to avoid heavy notation and to keep the derivations as clear as possible. This 
means that, when considering theories with both bosonic and fermionic generators, one cannot just copy the results from this paper, because we may have accidentally ordered the terms in a way that is allowed for the purely bosonic case, but not for the mixed case.

\section{The background field split}

As already explained in the introduction we will be dealing with a gauge theory in the presence of a boundary. At this boundary one must choose certain boundary conditions and the obvious one is to require that the boundary will be invariant under a subgroup of the full local gauge group. Hence one has to distinguish between the transformations that leave the boundary invariant and the transformations that act in the bulk, which will be integrated over in the path integral. This can be done systematically by first performing a background field split where the background refers to the boundary configuration extended into the bulk. The quantum fields are then viewed as fluctuations about this background and will eventually be integrated over in a path integral. At the boundary the quantum fields will vanish, but for the moment we refrain from discussing the details of these boundary conditions. For simplicity we restrict ourselves again to bosonic fields and transformation parameters.

To set up the background field split, let us consider a gauge theory with fields generically denoted by $\phi^{i}$, which are decomposed into background fields $\stackrel{\circ}{\phi}^{i}$ and quantum fields $\widetilde{\phi}^{i}$. The latter are the fields that one has to integrate over in a path integral. This integration requires to make use of a standard quantization method such as BRST quantization. The most straightforward decomposition between background and quantum fields is

$$
\phi^{i}=\stackrel{\circ}{\phi}^{i}+\widetilde{\phi}^{i},
$$

but in specific cases one may prefer to employ more sophisticated decompositions. Eventually the background fields are fixed at the boundary of the space and they are continued into the bulk. We assume that the precise continuation is not important because the deviation from their value in the bulk is characterized by the quantum fields which eventually will be integrated out. The gauge transformations are as specified in (2.1) and they can correspondingly be decomposed in two different ways. The background transformations $\AA^{\circ}$ take the form,

$$
\stackrel{\circ}{\delta}^{i}=R\left(\stackrel{\circ}{\phi}^{i}{ }_{\alpha} \stackrel{\circ}{\xi}^{\alpha}, \quad \stackrel{\circ}{\delta} \widetilde{\phi}^{i}=\Delta R(\dot{\phi}, \widetilde{\phi})^{i}{ }_{\alpha} \stackrel{\circ}{\xi}^{\alpha},\right.
$$

where $\Delta R(\dot{\phi}, \widetilde{\phi})^{i}{ }_{\alpha} \equiv R(\dot{\phi}+\widetilde{\phi})^{i}{ }_{\alpha}-R\left({ }_{\phi}\right)^{i}{ }_{\alpha}$. The gauge transformations $\tilde{\delta}$ that are relevant when integrating over the fields $\widetilde{\phi}^{i}$ must leave the background fields invariant and thus take the form,

$$
\tilde{\delta} \dot{\phi}^{i}=0, \quad \tilde{\delta} \widetilde{\phi}^{i}=R(\stackrel{\circ}{\phi}+\widetilde{\phi})^{i}{ }_{\alpha} \xi^{\alpha},
$$

and in the following we will keep referring to them as quantum transformations.

We start by considering the commutators of the quantum and background transformations acting on the background fields. For the background fields $\dot{\phi}^{i}$ a straightforward 
calculation yields

$$
\begin{aligned}
& {\left[\tilde{\delta}\left(\xi_{1}\right) \tilde{\delta}\left(\xi_{2}\right)-(1 \leftrightarrow 2)\right] \stackrel{\circ}{\phi}^{i}=0,} \\
& {[\stackrel{\circ}{\delta}(\dot{\xi}) \tilde{\delta}(\xi)-\tilde{\delta}(\xi) \stackrel{\circ}{\delta}(\stackrel{\circ}{\xi})] \stackrel{\circ}{\phi}^{i}=0,}
\end{aligned}
$$

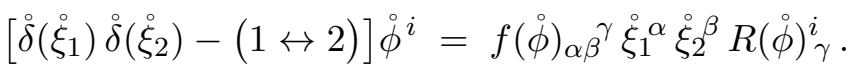

Subsequently one determines the same commutators, but now acting on the quantum fields,

$$
\begin{aligned}
& {\left[\tilde{\delta}\left(\xi_{1}\right) \tilde{\delta}\left(\xi_{2}\right)-(1 \leftrightarrow 2)\right] \widetilde{\phi}^{i}=f(\dot{\phi}+\widetilde{\phi})_{\alpha \beta^{\gamma}} \xi_{1}^{\alpha} \xi_{2}^{\beta} R(\dot{\phi}+\widetilde{\phi})_{\gamma}^{i},} \\
& {[\stackrel{\delta}{\delta}(\dot{\xi}) \tilde{\delta}(\xi)-\tilde{\delta}(\xi) \dot{\delta}(\dot{\xi})] \widetilde{\phi}^{i}=f(\stackrel{\dot{\phi}}{+}+\widetilde{\phi})_{\alpha \beta} \dot{\beta}^{\alpha} \dot{\xi}^{\beta} R(\dot{\phi}+\widetilde{\phi})_{\gamma}^{i},} \\
& {\left[\stackrel{\circ}{\delta}\left(\dot{\xi}_{1}\right) \stackrel{\circ}{\delta}\left(\dot{\xi}_{2}\right)-(1 \leftrightarrow 2)\right] \widetilde{\phi}^{i}=f(\stackrel{\circ}{\phi})_{\alpha \beta}{ }^{\gamma} \dot{\xi}_{1}^{\alpha} \dot{\xi}_{2}^{\beta} \Delta R(\dot{\phi}, \widetilde{\phi})_{\gamma}^{i}} \\
& +[f(\phi)-f(\dot{\phi})]_{\alpha \beta}^{\gamma} \stackrel{\circ}{\xi}_{1}^{\alpha} \dot{ }_{2}^{\beta} R(\stackrel{\circ}{\phi}+\widetilde{\phi})_{\gamma}^{i} \text {. }
\end{aligned}
$$

It is clear that the combined quantum and background transformations generate a closed algebra on $\dot{\phi}^{i}$ and $\widetilde{\phi}^{i}$. Its global structure has the following form,

$$
[\stackrel{\delta}{\delta}, \stackrel{\circ}{\delta}]=\stackrel{\delta}{\delta}+\widetilde{\delta}, \quad[\stackrel{\delta}{\delta}, \widetilde{\delta}]=\widetilde{\delta}, \quad[\widetilde{\delta}, \widetilde{\delta}]=\widetilde{\delta}
$$

When the algebra is soft, meaning that the structure 'constants' depend on the fields, then the background transformation will not form a subgroup. However, the closure of the full algebra remains unaffected.

Therefore we can construct a BRST complex by introducing two sets of ghosts, $\check{c}^{\alpha}$ and $c^{\alpha}$, corresponding to the background and the quantum transformations, respectively. Having introduced these variables, it is then straightforward to define the BRST transformations, which will eventually give rise to a nilpotent BRST charge. The BRST transformation on the fields $\dot{\phi}^{i}$ and $\widetilde{\phi}^{i}$ follows upon substituting $\xi^{\alpha}=\Lambda c^{\alpha}$ and $\dot{\xi}^{\alpha}=\Lambda \AA^{\alpha}$. The result reads as follows,

$$
\begin{aligned}
\delta_{\mathrm{brst}} \dot{\phi}^{i} & =R(\stackrel{\circ}{\phi})_{\alpha}^{i} \Lambda \stackrel{c}{\alpha}^{\alpha}, \\
\delta_{\mathrm{brst}} \widetilde{\phi}^{i} & =R(\dot{\phi}+\widetilde{\phi})_{\alpha}^{i} \Lambda\left(c^{\alpha}+\stackrel{\circ}{c}^{\alpha}\right)-R(\stackrel{\circ}{\phi})_{\alpha}^{i} \Lambda \dot{c}^{\alpha} .
\end{aligned}
$$

Here and in the remainder of this paper we will take into account that the theory contains both commuting and anti-commuting fields and gauge parameters. As it turns out the corresponding changes are rather minimal. As before the BRST transformations of the ghost fields follow straightforwardly from the commutation relations given in (3.4) and (3.5) and yield

$$
\begin{aligned}
& \delta_{\text {brst }} \stackrel{\circ}{c}^{\gamma}=\frac{1}{2} f(\stackrel{\circ}{\phi})_{\alpha \beta} \beta^{\gamma} \stackrel{\circ}{\alpha}^{\alpha} \Lambda \stackrel{\circ}{c}^{\beta},
\end{aligned}
$$

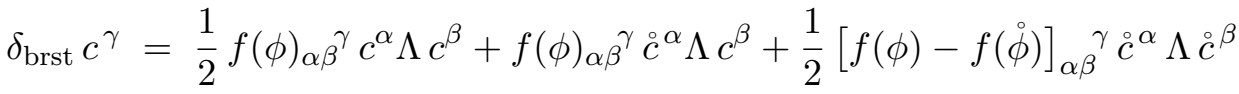

$$
\begin{aligned}
& =\frac{1}{2} f(\phi)_{\alpha \beta^{\gamma}}(c+\stackrel{\circ}{c})^{\alpha} \Lambda(c+\stackrel{\circ}{c})^{\beta}-\frac{1}{2} f(\stackrel{\circ}{\phi})_{\alpha \beta^{\gamma}} \stackrel{\circ}{c}^{\alpha} \Lambda \stackrel{\circ}{c^{\beta}} \text {, }
\end{aligned}
$$


where $\phi^{i}=\dot{\phi}^{i}+\widetilde{\phi}^{i}$. An interesting observation is that (3.8) leads to

$$
\delta_{\text {brst }}(c+\stackrel{o}{c})^{\gamma}=\frac{1}{2} f(\phi)_{\alpha \beta^{\gamma}}(c+\stackrel{\circ}{c})^{\alpha} \Lambda(c+\stackrel{\circ}{c})^{\beta},
$$

which confirms the consistency of splitting the ghosts into background ghosts $\dot{c}^{\alpha}$ and quantum ghosts $c^{\alpha}$, even in the case that the gauge algebra is soft! Note that the anti-ghosts $b_{\alpha}$ and the Lagrange multiplier fields $B_{\alpha}$ should be regarded as quantum fields, so that their BRST transformations remain unchanged and are given by

$$
\delta_{\text {brst }} b_{\alpha}=\Lambda B_{\alpha}, \quad \delta_{\text {brst }} B_{\alpha}=0 .
$$

The closure of the underlying gauge algebra expressed by the closure relations (3.4) and (3.5) now guarantees that the BRST charge is nilpotent, which can also be verified by explicit calculation,

$$
\delta_{\mathrm{brst}}^{2}=0 .
$$

The corresponding BRST invariant action is a generalization of (2.9). However, in this case one introduces only anti-ghosts $b_{\alpha}$ and Lagrange multipliers $B_{\alpha}$ associated with the quantum fields; for the background fields there will be no gauge-fixing terms. The quantum action then takes the form,

$$
\begin{aligned}
& S_{\text {brst }}\left[\widetilde{\phi}^{i}, c^{\alpha}, b_{\alpha}, B_{\alpha} ; \stackrel{\circ}{\phi}^{i}, \stackrel{\circ}{c}^{\alpha}\right]=\int \mathrm{d}^{n} x\left[\mathcal{L}^{\text {class }}(\stackrel{\circ}{\phi}+\widetilde{\phi})+B_{\alpha} F(\stackrel{\circ}{\phi}, \widetilde{\phi})^{\alpha}\right. \\
& -(-)^{\epsilon_{\alpha}+\epsilon_{\beta}+\epsilon_{j}} b_{\alpha} R(\dot{\phi}+\widetilde{\phi})^{j}{ }_{\beta}(c+\stackrel{\circ}{c})^{\beta} \tilde{\partial}_{j} F(\dot{\phi}, \widetilde{\phi})^{\alpha}
\end{aligned}
$$

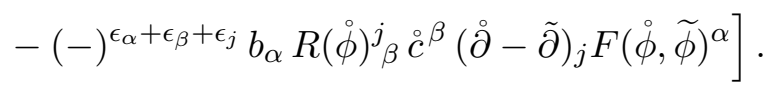

With suitable boundary conditions this is a BRST invariant functional of both the quantum and the background fields. Here we have assumed that the fields live in an $n$-dimensional space, and $\stackrel{\partial}{\partial}_{j}$ and $\widetilde{\partial}_{j}$ denote the derivatives with respect to $\dot{\phi}^{j}$ and $\widetilde{\phi}^{j}$, respectively. In the above equation they are defined as left-derivatives. Furthermore the gauge conditions $F^{\alpha}$ should be non-singular, meaning that $F(\dot{\phi}, \widetilde{\phi})^{\alpha}=0$ must fix the values of the quantum fields $\widetilde{\phi}^{i}$. Finally we observe that the ghosts $c^{\alpha}$ and $i^{\alpha}$ carry ghost number +1 , whereas the anti-ghosts $b_{\alpha}$ carry ghost number -1 , so that the action (3.12) carries zero ghost number.

The next step is to consider a functional integral over the quantum fields $\widetilde{\phi}^{i}$ and $c^{\alpha}$, $b_{\alpha}$ and $B_{\alpha}$,

$$
Z[\stackrel{\phi}{\phi}]=\int \mathcal{D} \widetilde{\phi}^{i} \mathcal{D} c^{\alpha} \mathcal{D} b_{\alpha} \mathcal{D} B_{\alpha} \exp \left[S_{\mathrm{brst}}\left[\widetilde{\phi}^{i}, c^{\alpha}, b_{\alpha}, B_{\alpha} ; \stackrel{\phi}{\phi}^{i}, \stackrel{\circ}{c}^{\alpha}\right]\right] .
$$

One can show that the restricted functional integration measure is BRST invariant under the same conditions as the full functional integral without background field splitting, namely

$$
\partial_{i} R(\phi)_{\alpha}^{i}=0, \quad f(\phi)_{\alpha \beta}{ }^{\beta}=0 .
$$

Since the indices on the fields include their space-time arguments, these two expressions are proportional to $\delta^{n}(0)$, where $\delta^{n}(x)$ is an $n$-dimensional delta function. Consequently 
they are ill-defined. This is a known complication, which has been studied in the past (see, for instance, $[27,28]$ ). On the basis of that we will assume from now on that the path integral in (3.13) is indeed fully consistent with regard to BRST transformations. Note that the action may still contain additional terms that depend exclusively on $B_{\alpha}$, because this field is BRST invariant. Irrespective of this, the integration measure for the fields $b_{\alpha}$ and $B_{\alpha}$ is BRST invariant by itself, so that no further modifications are required.

As already anticipated in the notation, the path integral $Z[\dot{\phi}]$ does not depend on the background ghosts. This follows directly from the observation that the right-hand side carries zero ghost number. Indeed, one can easily verify that the terms in (3.12) that are proportional to $b_{\alpha} \stackrel{\circ}{ }^{\beta}$ will not contribute to the functional integral. We have thus established that

$$
\delta_{\mathrm{brst}} Z[\stackrel{\circ}{\phi}]=\frac{\partial Z[\stackrel{\circ}{\phi}]}{\partial \dot{\circ}^{i}} R(\stackrel{\circ}{\phi})_{\alpha}^{i} \Lambda \stackrel{\circ}{c}^{\alpha},
$$

so that the functional integral is fully BRST invariant when the background specified by the fields $\dot{\phi}^{i}$ is invariant. Clearly the background ghosts only play an ancillary role here as the parameters that specify the background transformations. The existence of a consistent BRST complex that involves both quantum and background fields with corresponding ghost fields is a non-trivial result. It is remarkable that this result also holds for theories with a soft gauge algebra, where the structure constants depend on the fields.

To prove that the path integral (3.13) does not depend on the gauge condition, we first extend it by including external sources coupling to single fields or to composite operators. In this way one obtains a generating functional for Green's functions in a particular gauge, which can be used to derive BRST Ward identities. Hence we include an exponential factor with a variety of external sources into the integrand of the path integral $(3.13){ }^{3}$

$$
\exp \int \mathrm{d}^{n} x\left[J_{b}^{\alpha}(x) b_{\alpha}(x)+\widetilde{J}_{i}(x) \widetilde{\phi}^{i}(x)+J_{\alpha}^{c}(x) c^{\alpha}(x)+J_{B}^{\alpha}(x) B_{\alpha}(x)+\cdots\right] .
$$

The expansion of the path integral in terms of the external sources defines the corresponding Green's functions. Shifting the fields in (3.16) by the BRST-transformed fields leads to a rearrangement of Green's functions, while, on the other hand, the extra terms can be eliminated by making use of the fact that $S_{\text {brst }}$ and the integration measure of the functional integral is BRST invariant, up to the transformations of the background fields. In this way one thus obtains the Ward identities between Green's functions. There is an implicit assumption here, namely that BRST symmetry is manifest and not realized in a spontaneously broken way. If that were not the case, then the invariant action would contribute to the Ward identities in the form of the divergence of the BRST Noether current.

Let us now derive two Ward identities and discuss their consequences. In the first one we put all sources to zero with the exception of $J_{b}^{\alpha}$. The Ward identity then takes the form

$$
\int \mathcal{D} \widetilde{\phi}^{i} \mathcal{D} c^{\alpha} \mathcal{D} b_{\alpha} \mathcal{D} B_{\alpha} \exp \left[S_{\text {brst }}+\int \mathrm{d}^{n} y J_{b}^{\gamma}(y) b_{\gamma}(y)\right] \int \mathrm{d}^{n} x J_{b}^{\alpha}(x) \Lambda B_{\alpha}(x)=0,
$$

\footnotetext{
${ }^{3}$ External sources coupling to background fields are not revelvant here as the path integral does not involve an integration over these fields.
} 
where we used the BRST variation of the anti-ghost field. Only the term linear in $J_{b}^{\alpha}$ can give a non-zero contribution, because the ghost fields in the action are all paired with anti-ghost fields. Since the source is not subject to any restriction it thus follows that the expectation value of $B_{\alpha}$ must vanish, i.e.

$$
\int \mathcal{D} \widetilde{\phi}^{i} \mathcal{D} c^{\alpha} \mathcal{D} b_{\alpha} \mathcal{D} B_{\alpha} \exp \left[S_{\text {brst }}\right] B_{\alpha}(x)=0 .
$$

On the other hand, whether or not the expectation value of $B_{\alpha}$ will vanish is eventually a dynamical question that depends on the details of the action $S_{\mathrm{brst}}$. When the expectation value does not vanish, the BRST symmetry will be realized in a spontaneously broken way in view of the fact that the expectation value of $\delta_{\text {brst }} b_{\alpha}$ will not vanish. In that case the Ward identity will receive extra contributions as we already indicated previously. However, it is obvious that this option is of no physical interest, and one has to insist that BRST invariance is manifestly realized [26].

For the second Ward identity we keep the dependence on the source $J_{b}^{\alpha}$ but in addition we now consider a second source coupling to a composite operator $\Delta F(\widetilde{\phi}, \stackrel{\circ}{\phi})^{\beta}$. The terms of higher order in $J_{b}^{\alpha}$ will not contribute, just as in the previous case, and we will restrict ourselves to the first-order contribution in the composite operator. By differentiating with respect to the two external sources one thus derives the following Ward identity,

$$
\begin{aligned}
& \int \mathcal{D} \widetilde{\phi}^{i} \mathcal{D} c^{\alpha} \mathcal{D} b_{\alpha} \mathcal{D} B_{\alpha} \exp \left[S_{\text {brst }}\right] \\
& \quad \times\left[\Delta F(\widetilde{\phi}, \stackrel{\circ}{\phi})^{\beta}(y) \Lambda B_{\alpha}(x)+\delta_{\text {brst }} \Delta F(\widetilde{\phi}, \stackrel{\circ}{\phi})^{\beta}(y) b_{\alpha}(x)\right]=0 .
\end{aligned}
$$

Upon integrating this result over $x$ and $y$ with a delta function $\delta^{n}(x-y)$ and contracting the indices with $\delta^{\alpha}{ }_{\beta}$, one recognizes that this result is precisely the original result (3.13) for $Z[\stackrel{\circ}{\phi}]$ but now with a gauge-fixing term equal to $F(\widetilde{\phi}, \dot{\phi})^{\alpha}+\Delta F(\widetilde{\phi}, \stackrel{\circ}{\phi})^{\alpha}$, expanded to first order in $\Delta F^{\alpha}$. This proves that $Z[\stackrel{\circ}{\phi}]$ is independent of the choice of the gauge condition. ${ }^{4}$

An interesting observation in view of what will be discussed later, is that the gauge independence is not affected when including extra terms in the action that are BRST exact, i.e. terms that can be written as the BRST variation of functions of the fields $\widetilde{\phi}^{i}$ and $\dot{\phi}^{i}$. In the specific context of BRST quantization this observation is not particularly useful, as these terms will violate ghost number conservation. Only the gauge-fixing term, which is also BRST exact, will preserve ghost number by virtue of the presence of the anti-ghost field.

\section{Equivariant cohomology}

In the previous section we have presented a consistent background field split in which the original fields have been decomposed into background and quantum fields, denoted by $\dot{\phi}^{i}$ and $\widetilde{\phi}^{i}$, respectively, thus doubling all the fields. Correspondingly we have also doubled

\footnotetext{
${ }^{4}$ This particular argument is a slight generalization of the analysis presented in [29], which was used to derive the gauge independence of the S-matrix in gauge theories with quadratic gauge fixing (where BRST is not nilpotent on the anti-ghost fields $b_{\alpha}$ ).
} 
the gauge transformations in terms of background and quantum gauge transformations, and we have shown that they can be incorporated consistently into an extended BRST complex. This extension can be given irrespective of whether the gauge algebra is soft or not. We only used that the gauge transformations close off shell.

This particular set-up was proposed in order to deal with gauge theories in the presence of a boundary. The boundary values of the original fields, which will be motivated primarily by physical considerations, are carried by the background fields $\dot{\phi}^{i}$ that will be smoothly continued into the bulk. The quantum fields $\widetilde{\phi}^{i}$, on the other hand, describe the fluctuations in the bulk about the selected background fields; obviously the quantum fields must therefore vanish at the boundary. Their fluctuations will eventually be integrated over in a suitable path integral as was shown in the previous section.

The background fields $\dot{\phi}^{i}$ will typically be invariant under an isometry group that is a subgroup of the full group of background transformations. In the continuation of the background fields into the bulk, the isometry group has to remain manifest. The background ghosts should then be restricted to take their values in the isometry algebra. All this implies that the BRST transformations on the background fields are necessarily constrained to vanish,

$$
\delta_{\mathrm{brst}} \stackrel{\phi}{ }^{i}=R(\stackrel{\circ}{\phi})_{\alpha}^{i} \Lambda \stackrel{\circ}{\alpha}^{\alpha}=0 .
$$

Consequently the background ghosts $i^{\alpha}$, which play the role of symmetry parameters associated to the background transformations, should vanish with the exception of those that parametrize the isometry group of the background field configuration. Since the isometry group is a subgroup of the full background symmetry group, this ensures that the above restriction can be imposed consistently. Here we are implicitly assuming that the isometry group is defined for the global background field configuration (i.e. also in the bulk), which poses a restriction on how the background fields are continued into the bulk. The non-vanishing background ghosts $\stackrel{\circ}{c}^{\alpha}$ that parametrize the isometry group will in general be subject to differential constraints that are implied by the appropriate Killing equations associated with the background isometries. Under these conditions the structure constants of the background isometry algebra follow obviously from the original structure constants $f(\dot{\phi})_{\alpha \beta} \gamma$ upon considering the explicit embedding of the isometry group into the full background symmetry group. As far as the BRST transformations are concerned the possible field-dependence of $f(\stackrel{\circ}{\phi})_{\alpha \beta}{ }^{\gamma}$ is not relevant in view of the constraint (4.1). Because of this constraint the BRST transformations of the quantum fields $\widetilde{\phi}^{i}$ simplify and take the form

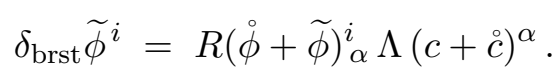

Note that one can subsequently consider a possible subalgebra of the isometry algebra by further restricting the number of background ghosts. In the subsequent discussion it will be important that some of the background ghosts remain present and will generate a non-trivial subgroup of the background isometries, so that (4.1) remains valid.

Let us now continue and consider the BRST transformation on the background ghosts,

$$
\delta_{\mathrm{brst}} \stackrel{\circ}{c}^{\alpha}=\frac{1}{2} f(\stackrel{\circ}{\phi})_{\beta \gamma}^{\alpha} \stackrel{\circ}{c}^{\beta} \Lambda \stackrel{\circ}{c}^{\gamma} .
$$


This variation is consistent with the reduction of the background ghosts to the isometry algebra. Therefore there is no need for introducing any additional constraints on

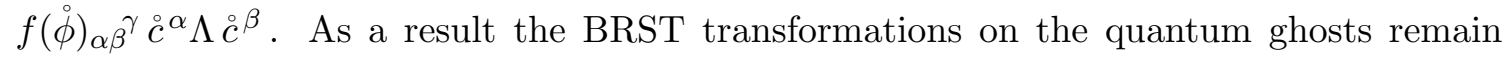
unchanged,

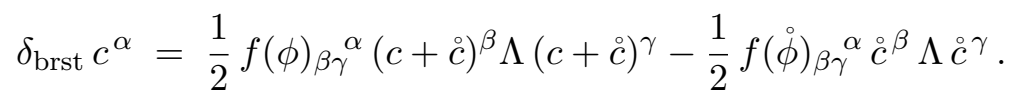

It is now straightforward to verify that the BRST symmetry is still nilpotent. As before this requires to use the Jacobi identity (2.4), which simplifies for the background structure constants because $\dot{\phi}^{i}$ is now BRST invariant. Furthermore it follows from (3.15) that the path integral (3.13) is BRST invariant as well.

None of the quantum fields are constrained, and therefore they will appear as before in the functional integral (3.13); this integral now involves a coupling to a restricted set of background fields, $\dot{\phi}^{i}$ and $\stackrel{\circ}{c}^{\alpha}$, but nevertheless it remains well-defined, also in view of the fact that the functional integral did not include an integration over the background fields and ghosts.

Until now we did not change the original BRST algebra, but rather we adopted a special field representation by requiring that the background fields $\dot{\phi}^{i}$ were BRST invariant. This implied that the background ghosts $\dot{c}^{\alpha}$ had to be restricted to take their values in the corresponding isometry subalgebra. As a next step we now introduce a deformation of the BRST algebra by imposing the condition that also background ghosts will remain invariant under the algebra, without implying that the right-hand side of (4.3) must vanish. Upon imposing this deformation both the background fields and the background ghosts will thus remain invariant, while the transformations of the quantum fields are unchanged. We denote the resulting variations by $\delta_{\text {eq }}$, which take the following form,

$$
\begin{aligned}
& \delta_{\text {eq }} \dot{\phi}^{i}=0, \quad \delta_{\text {eq }} \stackrel{\circ}{\alpha}^{\alpha}=0, \\
& \delta_{\mathrm{eq}} \widetilde{\phi}^{i}=R(\stackrel{\circ}{\phi}+\widetilde{\phi})_{\alpha}^{i} \Lambda\left(c^{\alpha}+\dot{c}^{\alpha}\right),
\end{aligned}
$$

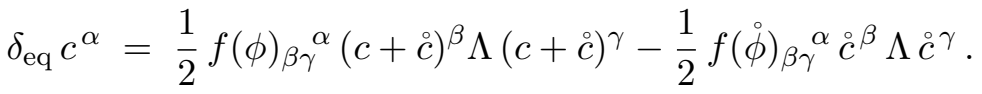

As the reader can verify these transformations are no longer nilpotent. Instead they define an equivariant map. The relevant relations, which follow again by making use of the closure relation (2.3) and the Jacobi identity (2.4), are

$$
\delta_{\mathrm{eq}}^{2}=\delta_{\dot{\xi}}, \quad\left[\delta_{\mathrm{eq}}, \delta_{\dot{\xi}}\right]=0 .
$$

The new transformation $\delta_{\xi}$ acts on the quantum fields according to

$$
\begin{aligned}
& \delta_{\dot{\xi}} \widetilde{\phi}^{i}=R(\dot{\phi}+\widetilde{\phi})_{\alpha}^{i} \stackrel{\circ}{\xi}^{\alpha}, \\
& \delta_{\dot{\xi}} c^{\alpha}=f(\dot{\phi}+\widetilde{\phi})_{\beta \gamma}{ }^{\alpha}(c+\stackrel{\circ}{c})^{\beta} \stackrel{\circ}{\xi}^{\gamma} \text {, }
\end{aligned}
$$

with the transformation parameter $\stackrel{\circ}{\xi}^{\alpha}$ equal to

$$
\stackrel{\circ}{\xi}^{\alpha} \equiv \Lambda_{[2} f(\stackrel{\circ}{\phi})_{\beta \gamma}{ }^{\alpha} \stackrel{\circ}{c}^{\beta} \Lambda_{1]} \stackrel{\circ}{c}^{\gamma} .
$$


Note that the $\stackrel{\circ}{\xi}^{\alpha}$ take their values in the isometry algebra. The background fields and ghosts are obviously invariant under $\delta_{\xi}$.

The equivariant algebra (4.6) must also be defined on the anti-ghosts and the Lagrange multiplier fields. Assuming that $\delta_{\text {eq }} b_{\alpha}$ coincides with $\delta_{\text {brst }} b_{\alpha}$, one deduces the form of $\delta_{\text {eq }} B_{\alpha}$,

$$
\begin{aligned}
\delta_{\text {eq }} b_{\alpha} & =\Lambda B_{\alpha}, \\
\delta_{\text {eq }} B_{\alpha} & =\frac{1}{2} f(\stackrel{\circ}{\phi})_{\delta \varepsilon}^{\beta}{ }^{\circ}{ }^{\delta} \Lambda \stackrel{\circ}{c}^{\varepsilon} f(\stackrel{\circ}{\phi})_{\alpha \beta}{ }^{\gamma} b_{\gamma} .
\end{aligned}
$$

The action of $\delta_{\xi}$ on both $b_{\alpha}$ and $B_{\alpha}$ then follows from imposing the algebra (4.6). The result is

$$
\begin{aligned}
& \delta_{\xi} b_{\alpha}=\stackrel{\circ}{\xi}^{\beta} f(\stackrel{\circ}{\phi})_{\alpha \beta}{ }^{\gamma} b_{\gamma}, \\
& \delta_{\xi} B_{\alpha}=\stackrel{\circ}{\xi}^{\beta} f(\stackrel{\circ}{\phi})_{\alpha \beta}{ }^{\gamma} B_{\gamma} \text {. }
\end{aligned}
$$

The variations $\delta_{\text {eq }}$ defined in (4.5), (4.9), and $\delta_{\xi}$ defined in (4.7), (4.10), have a well-defined ghost number equal to 1 and 2 , respectively.

One expects that the boundary should be invariant under both $\delta_{\text {eq }}$ and $\delta_{\xi}$. This is directly confirmed by applying the generators of the equivariant algebra on the quantum fields $\widetilde{\phi}^{i}, c^{\alpha}, b_{\alpha}$, and $B_{\alpha}$, which themselves vanish at the boundary. Indeed it is easy to verify that their variations under $\delta_{\text {eq }}$ and $\delta_{\xi}$ vanish also at the boundary by virtue of (4.1) and the Jacobi identity for the structure constants of the background isometry algebra. Note that this is a local result. The global boundary can only be invariant provided it contains no singular points. Especially for spaces of Minkowskian signature this may be an issue. Here we will ignore this subtlety and assume that the boundary is indeed regular.

The above considerations provide us with a special background isometry $\delta_{\xi}$ obeying $\delta_{\xi}=\delta_{\text {eq }}^{2}$ that acts on all the quantum fields while leaving the background fields and ghosts invariant. Hence the quantum fields do transform under the isometries of the background and their transformation rules are specified by the terms in $\delta_{\text {eq }}$ proportional to the background ghosts $\AA^{\alpha} .^{5}$

We already concluded that the functional integral $Z[\stackrel{\circ}{\phi}]$ in $(3.13)$ is a BRST invariant functional of the background fields $\dot{\phi}^{i}$, so that the BRST invariance of $Z[\stackrel{\phi}{\phi}]$ seems to imply its invariance under $\delta_{\text {eq }}$, and therefore also under $\delta_{\xi}$. This expectation is indeed confirmed by explicit computations. According to (4.5) and (4.9) the operator $\delta_{\text {eq }}$ differs from its nilpotent ancestor $\delta_{\text {brst }}$ only in its action on the background ghosts $\stackrel{\circ}{c}^{\alpha}$ and the Lagrange multiplier fields $B_{\alpha}$. Bearing in mind that $\dot{\phi}^{i}$ is invariant, $\delta_{\text {eq }}(\dot{\phi}+\widetilde{\phi})^{i}$ is identical to the original BRST transformation so that the classical Lagrangian $\mathcal{L}^{\text {class }}$ is also invariant under $\delta_{\text {eq. }}$. However, the gauge-fixing term $\mathcal{L}^{\text {g.f. }}$ does explicitly depend on $\stackrel{\circ}{\alpha}^{\alpha}$ and $B_{\alpha}$, so let us take a closer look. First we note that the last line present in (3.12) will now vanish by virtue of (4.1). Therefore the gauge-fixing term that appears in (3.12) becomes identical to

\footnotetext{
${ }^{5}$ Alternative ways of modifying the BRST algebra have been described in the literature (see e.g. [30-33]), but they are conceptually different from the present proposal.
} 
(for convenience we specialize again to commuting gauge transformations and commuting fields $\left.\stackrel{\circ}{\phi}^{i}, \widetilde{\phi}^{i}\right)$,

$$
\mathcal{L}^{\text {g.f. }}=B_{\alpha} F(\dot{\phi}, \widetilde{\phi})^{\alpha}-b_{\alpha} \delta_{\mathrm{eq}} F(\dot{\phi}, \widetilde{\phi})^{\alpha} \text {. }
$$

It is now clear that $\delta_{\text {eq }} \mathcal{L}^{\text {g.f. }}$ does not vanish. Instead it will be proportional to $b_{\alpha}$, resulting from the variation of $B_{\alpha}$ given in (4.9) and from the fact that $\delta_{\mathrm{eq}}{ }^{2} F^{\alpha}$ is non-vanishing and equal to $\delta_{\xi} F^{\alpha}$. Not surprisingly these terms combine into the $\delta_{\xi}$ variation of the gauge fermion $b_{\alpha} F(\dot{\phi}, \widetilde{\phi})^{\alpha}$. Therefore we conclude that the action

$$
S_{\text {eq }}\left[\widetilde{\phi}^{i}, c^{\alpha}, b_{\alpha}, B_{\alpha} ; \stackrel{\circ}{\phi}^{i}, \stackrel{\circ}{c}^{\alpha}\right]=\int \mathrm{d}^{n} x\left[\mathcal{L}^{\text {class }}(\dot{\phi}+\widetilde{\phi})+\partial_{\Lambda} \delta_{\text {eq }}\left[b_{\alpha} F(\stackrel{\circ}{\phi}, \widetilde{\phi})^{\alpha}\right]\right]
$$

satisfies

$$
\delta_{\text {eq }} S_{\text {eq }}=\delta_{\dot{\xi}} \int \mathrm{d}^{n} x\left[b_{\alpha} F(\dot{\phi}, \widetilde{\phi})^{\alpha}\right]
$$

where we wrote the variation $\delta_{\xi}$ outside the integral in view of the fact that the boundary is invariant.

The functional integral $Z[\stackrel{\phi}{\phi}]$ can now also be written as

$$
Z[\stackrel{\circ}{\phi}]=\int \mathcal{D} \widetilde{\phi}^{i} \mathcal{D} c^{\alpha} \mathcal{D} b_{\alpha} \mathcal{D} B_{\alpha} \exp \left[S_{\text {eq }}\left[\widetilde{\phi}^{i}, c^{\alpha}, b_{\alpha}, B_{\alpha} ; \stackrel{\circ}{\phi}^{i}, \stackrel{\circ}{c}^{\alpha}\right]\right],
$$

because the right-hand side of (4.13) will cancel under the functional integral over the ghost fields for the simple reason that it generates terms proportional to the anti-ghosts without corresponding ghosts. Furthermore the functional integration measure is also invariant under $\delta_{\text {eq }}$ since the contributions of the variation from the ghosts and quantum fields vanish by our earlier assumptions (3.14), and the transformations of the anti-ghosts $b_{\alpha}$ and Lagrange multipliers $B_{\alpha}$ have a trivial Jacobian. Putting these facts together, we reach the conclusion that indeed $\delta_{\text {eq }}$ is a symmetry of the functional integral (3.13), i.e.,

$$
\delta_{\text {eq }} Z[\stackrel{\circ}{\phi}]=0 .
$$

Although there was no need for requiring that (4.13) must vanish in order to prove that $Z[\phi]$ is invariant under $\delta_{\text {eq }}$, we should point out that the situation will be different when considering deformations of the integrand. Therefore we will assume henceforth that the background ghosts are chosen such that the background isometry $\delta_{\xi}$ is compact, so that integrals as in (4.13) will generically vanish.

Finally we consider the dependence of the functional integral on the gauge condition. As it turns out, one can use the same strategy as followed at the end of section 3 to show that the functional integral is gauge independent. One can also verify that deformations of the functional integral associated with $\delta_{\text {eq }}$-exact terms will leave the gauge independence unaffected, provided that $\delta_{\xi}$ is compact. In this respect, the situation is similar to that of the BRST complex, discussed in section 3.

\section{Localization of the functional integral}

The formulation developed in the previous sections seems ideally suited for applying localization in a large class of theories that admit local supersymmetry transformations as part 
of their gauge algebra. In particular, we are now able to generalize previous applications of localization, which so far have mainly been confined to gauge theories with rigid supersymmetry, to theories of supergravity. To do so, consider the functional integral (4.14) where $\mathcal{L}^{\text {class }}$ is a supergravity Lagrangian. ${ }^{6}$ The formalism does not rely on the particular form of the classical Lagrangian, and we are able to discuss supergravity theories which also include higher-derivative couplings, such as those discussed in [34-36]. Observe that in an off-shell formulation of supergravity, the gauge-fixing described in sections 2 and 3 results in an equal number of bosonic and fermionic degrees of freedom. The manipulations described in section 4 will only affect the number of background fields and ghosts, so that the quantum fields will still comprise an equal number of bosonic and fermionic fields. This is a useful feature of the covariantly quantized off-shell theory that we will use below. Concerning the background fields and ghosts, we assume that the background isometries constitute a rigid superalgebra. The invariance under these isometries then allows one to consider a purely bosonic background.

Let us now turn to the localization strategy for evaluating (4.14). The main idea is to deform the functional integral to reach a convenient point in field space where we can evaluate it exactly by using semiclassical methods. Such a deformation $Z[\stackrel{\circ}{\phi}]=Z[\stackrel{\circ}{\phi} ; 0] \rightarrow$

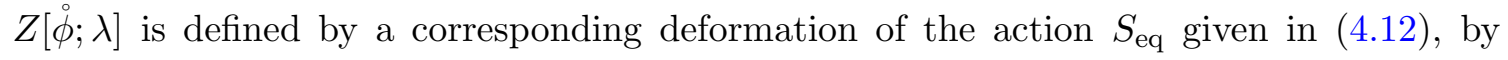
$S_{\text {eq }}=S(0) \rightarrow S(\lambda)=S(0)+\lambda \delta_{\text {eq }} \mathcal{V}$, where $\lambda$ is a real deformation parameter. The expression for $\mathcal{V}$ is chosen to satisfy $\delta_{\text {eq }}{ }^{2} \mathcal{V}=0$, so that the deformation is $\delta_{\text {eq }}$-exact and $\delta_{\dot{\xi}} \mathcal{V}=0$. Differentiating with respect to the parameter $\lambda$ pulls down a factor of $\delta_{\text {eq }} \mathcal{V}$ in the functional integral, so that we can write

$$
\frac{d}{d \lambda} Z[\stackrel{\circ}{\phi} ; \lambda]=\int \mathcal{D} \widetilde{\phi}^{i} \mathcal{D} c^{\alpha} \mathcal{D} b_{\alpha} \mathcal{D} B_{\alpha} \delta_{\text {eq }}\left[\mathcal{V} \exp \left[S_{\text {eq }}+\lambda \delta_{\text {eq }} \mathcal{V}\right]\right]
$$

Here we have used that $S_{\text {eq }}$ vanishes under the action of $\delta_{\text {eq }}$, based on the restriction that the background isometry $\delta_{\xi}$ should be compact (see the comment at the end of section 4 ).

Assuming that $\delta_{\text {eq }}$ can be represented as a differential operator in field space [37], we conclude that

$$
\frac{d}{d \lambda} Z[\dot{\phi} ; \lambda]=0
$$

It is important to mention that one of the conditions for localization is that the manifold on which the theory is defined is compact, which can only be achieved in the situations we will be considering by introducing a cut-off on the asymptotics, as is for instance done in AdS/CFT calculations. Our formalism enables us to consider such a boundary in a systematic way that is consistent with supersymmetry, but one still has to investigate whether sending the cut-off to infinity will not introduce any undesirable effects. Assuming that this is not the case, then $Z[\stackrel{\phi}{\phi} ; \lambda]$ will be independent of the deformation parameter.

An immediate consequence of the property (5.2) is that we can evaluate the original functional integral by taking the parameter $\lambda$ to be very large in order to reach a con-

\footnotetext{
${ }^{6}$ The functional integral in quantum field theory is, of course, only a formal physical concept that is not well-defined, especially not in quantum gravity, because of severe short-distance singularities. As in many supersymmetric theories, the hope is that supersymmetry holds at all scales, and that the formal procedure based on localization will be valid, irrespective of the serious complications in the perturbative context.
} 
venient point in field space. In this regime, $Z[\dot{\phi} ; \lambda]$ localizes to the critical points of the deformation $\delta_{\text {eq }} \mathcal{V}$. To explain how this limit works in detail, we make a convenient choice for the deformation by adopting the following definition ${ }^{7}$

$$
\mathcal{V}=\int \mathrm{d}^{n} x \sum_{\bar{\imath}} \sqrt{\stackrel{\circ}{g}} \bar{\psi}_{\bar{\imath}} \delta_{\text {eq }} \psi^{\bar{\imath}}
$$

where we have introduced a suitably chosen background space-time metric and the sum involves all fermion fields belonging to the quantum fields $\widetilde{\phi}^{i}$, with the notable exception of the ones already present in the gauge-fixing conditions used to quantize the fermionic gauge symmetries in the original functional integral. Correspondingly, we have split the index $i$ and denote the fermions entering $\mathcal{V}$ by an index $\bar{\imath}$. We also remind the reader that we have previously imposed the condition that $\delta_{\mathrm{eq}}{ }^{2} \mathcal{V}$ must vanish. Indeed

$$
\delta_{\text {eq }}^{2} \mathcal{V}=\delta_{\dot{\xi}} \int \mathrm{d}^{n} x \sum_{\bar{\imath}} \sqrt{\stackrel{\circ}{g}} \bar{\psi}_{\bar{\imath}} \delta_{\text {eq }} \psi^{\bar{\imath}}=0
$$

is satisfied based on the fact that the background isometry $\delta_{\xi}$ is compact. The deformed action corresponding to (5.3) now takes the form,

$$
\begin{aligned}
S(\lambda)= & S^{\text {class }}[\stackrel{\circ}{\phi}+\widetilde{\phi}]+\int \mathrm{d}^{n} x\left[B_{\alpha} F(\stackrel{\circ}{\phi}, \widetilde{\phi})^{\alpha}+(-)^{\epsilon_{\alpha}} b_{\alpha} \delta_{\mathrm{eq}} F(\stackrel{\circ}{\phi}, \widetilde{\phi})^{\alpha}\right] \\
& +\lambda \int \mathrm{d}^{n} x \sum_{\bar{\imath}} \sqrt{\stackrel{\circ}{g}}\left[\delta_{\mathrm{eq}} \bar{\psi}_{\bar{\imath}} \delta_{\mathrm{eq}} \psi^{\bar{\imath}}-\bar{\psi}_{\bar{\imath}} \delta_{\mathrm{eq}}^{2} \psi^{\bar{\imath}}\right] .
\end{aligned}
$$

It is important to stress that the above action is properly quantized and therefore free of gauge redundancies, owing to the presence of the gauge-fixing terms. As a result, all quantum corrections derived from it are well-defined for any value of the deformation parameter $\lambda$. We also remind the reader that the functional integral is independent of the gauge choices. This can be shown by following the same arguments as used at the end of section 3 in the context of BRST cohomology.

In the limit $\lambda \rightarrow \infty$ the critical points of the deformation dominate the functional integral. We assume that this critical locus is bosonic, i.e. we can set all the anti-commuting fields and ghosts to zero. The resulting localization manifold is

$$
\mathcal{M}=\left\{\delta_{\mathrm{eq}} \psi^{\bar{\imath}}=0 \text { for all fermions } \psi^{\bar{\imath}} \in \widetilde{\phi}^{i} / F(\stackrel{\circ}{\phi}, \widetilde{\phi})^{\alpha}=0\right\} \equiv\left\{t_{a}\right\},
$$

where the parameters $t_{a}$ are appropriately chosen coordinates on the solution set $\mathcal{M}$. As indicated, the localization manifold is subject to the bosonic gauge-fixing conditions which ensure that quantum corrections are properly quantized on $\mathcal{M}$, and (5.6) instructs us to

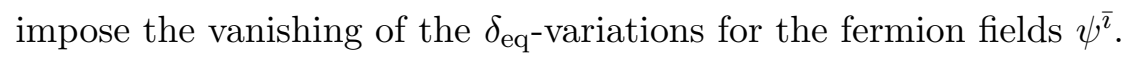

To appreciate what the consequences are of the conditions $\delta_{\text {eq }} \psi^{\bar{\imath}}=0$, we remind the reader that the purely bosonic terms of $\delta_{\text {eq }} \psi^{\bar{\imath}}$ take the form $R(\stackrel{\circ}{\phi}+\widetilde{\phi})^{\bar{\alpha}} \bar{\alpha}(\stackrel{\circ}{c}+c)^{\bar{\alpha}}$, where

\footnotetext{
${ }^{7}$ Here the bar on the fermions $\psi$ indicates an appropriate conjugation. The action of this conjugation on the fields of the theory is known to be subtle even in gauge theories with rigid supersymmetry, as there is always some tension between the reality conditions of fields and positive-definiteness of $\delta_{\text {eq }} \mathcal{V}$. The recent work [38] on Euclidean supergravity may help in clarifying this issue.
} 
the index $\bar{\alpha}$ refers to fermionic gauge parameters so that their corresponding ghosts are commuting fields. ${ }^{8}$ For a bosonic localization manifold the dependence of $R(\dot{\phi}+\widetilde{\phi})^{\bar{\imath}} \bar{\alpha}$ on the fermion fields is suppressed so that this manifold will involve the bosonic fields $\widetilde{\phi}^{\hat{\imath}}$, subject to gauge conditions, and $c^{\bar{\alpha}}$. Both types of fields must vanish on the boundary. The background fields $\dot{\phi}^{i}$ and $\dot{c}^{\alpha}$ are subject to the invariance condition (4.1). The background ghosts $\stackrel{\circ}{c}^{\alpha}$ that parametrize the background isometries must be restricted such that the square of the corresponding $\delta_{\text {eq }}$ variation yields a compact $\delta_{\xi}$ (cf. the discussion below (4.15)). The equations $R(\stackrel{\circ}{\phi}+\widetilde{\phi})^{\bar{\alpha}} \Lambda(\stackrel{\circ}{c}+c)^{\bar{\alpha}}=0$ then impose relations between the fields $\widetilde{\phi}^{\hat{\imath}}$ and $c^{\bar{\alpha}}$ that lead to the localization manifold. This manifold will be parametrized in terms of the independent coordinates $t_{a}$ that we have introduced in (5.6). Not surprisingly, the same type of equations are encountered when determining supersymmetric field configurations in classical supergravity, where the ghost fields are replaced by the parameters of the supersymmetry transformations. There are various ways to solve such equations, and we will discuss a specific application in the next section by way of an illustration.

The localization manifold $\mathcal{M}$ thus corresponds to the set of critical points of the deformation with certain values for the bosonic fields $\widetilde{\phi}^{\hat{\imath}}$ and $c^{\bar{\alpha}}$, which we denote by $\left.\widetilde{\phi}^{\hat{\imath}}(t)\right|_{\mathcal{M}}$ and $\left.c^{\bar{\alpha}}(t)\right|_{\mathcal{M}}$. Fluctuations around the localization manifold also contribute to the functional integral, and in the large- $\lambda$ limit the one-loop contribution becomes exact. To see this, we can expand the quantum fields as follows

$$
\widetilde{\phi}^{i}=\left.\widetilde{\phi}^{i}(t)\right|_{\mathcal{M}}+\frac{1}{\sqrt{\lambda}} \widetilde{\phi}^{i \prime}, \quad c^{\alpha}=\left.c^{\alpha}(t)\right|_{\mathcal{M}}+\frac{1}{\sqrt{\lambda}} c^{\alpha \prime},
$$

where the fermionic fields $\left.\widetilde{\phi}^{\overline{ }}(t)\right|_{\mathcal{M}}$ and $\left.c^{\hat{\alpha}}(t)\right|_{\mathcal{M}}$ vanish. As alluded to above, the anti-ghost

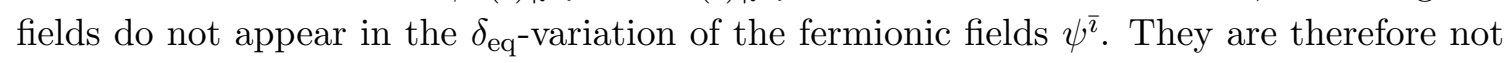
part of the localization manifold and should be regarded as quantum fluctuations. Since, as we stressed, the action (5.5) has no gauge degeneracy to begin with, we should also rescale the anti-ghost and Lagrange multiplier fields as

$$
b_{\alpha}=\sqrt{\lambda} b_{\alpha}{ }^{\prime}, \quad B_{\alpha}=\sqrt{\lambda} B_{\alpha}{ }^{\prime} .
$$

This ensures that the propagators for the fluctuations around $\mathcal{M}$ scale appropriately and remain well-defined. In this way, the gauge-fixing of the original undeformed functional integral naturally guarantees that the theory describing fields along the localization manifold as well as their fluctuations is also free of gauge redundancies.

With these definitions one can expand the action (5.5) according to (5.7) and (5.8), taking into account that the localization manifold is purely bosonic. The result is then equal to the classical action evaluated on the localization manifold and all the terms from the deformation and the gauge-fixing terms proportional to the anti-ghosts that are quadratic in the fluctuations $\widetilde{\phi}^{i \prime}, c^{\alpha^{\prime}}$ and $b_{\alpha}^{\prime}$, up to terms that vanish in the large- $\lambda$ limit. Integrating over these fluctuations then gives rise to the following result for the functional integral,

$$
Z[\stackrel{\circ}{\phi}]=\int_{\mathcal{M}} \mu(t) \mathrm{d} t_{a} \exp \left[S^{\text {class }}\left[\stackrel{\circ}{\phi}, \stackrel{\circ}{c} ; t_{a}\right]\right] Z_{1-\text { loop }}\left[\stackrel{\circ}{\phi}, \stackrel{\circ}{c} ; t_{a}\right]
$$

\footnotetext{
${ }^{8}$ We remind the reader that $\Lambda$ is only present to keep track of the relative signs between the contributions from fermionic and bosonic fields. When writing the various expressions explicitly in terms of fermionic and bosonic fields, the presence of $\Lambda$ can be avoided.
} 
where we have assumed the presence of a measure $\mu(t)$ induced by the embedding of the localization manifold into the field configuration space. This measure can in principle be evaluated from the explicit expressions for $\left.\widetilde{\phi}^{\imath}(t)\right|_{\mathcal{M}}$ and $\left.c^{\bar{\alpha}}(t)\right|_{\mathcal{M}}$.

The last term $Z_{1 \text {-loop }}\left[\dot{\phi}, \stackrel{\circ}{c} ; t_{a}\right]$ under the integral contains the semiclassical correction caused by the integration over the quantum fluctuations of the fields about the localization manifold,

$$
\begin{aligned}
Z_{\text {1-loop }}\left[\grave{\phi}, \stackrel{\circ}{c} ; t_{a}\right]=\int \mathcal{D}\left(\widetilde{\phi}^{i \prime}\right) & \mathcal{D}\left(c^{\alpha \prime}\right) \mathcal{D}\left(b_{\alpha}{ }^{\prime}\right) \mathcal{D}\left(B_{\alpha}{ }^{\prime}\right) \\
& \times\left.\exp \left[\delta_{\text {eq }}\left[\mathcal{V}+b_{\alpha}{ }^{\prime} F\left(\dot{\phi} ; t_{a}, \widetilde{\phi}^{\prime}\right)^{\alpha}\right]\right]\right|_{\text {quad. }} .
\end{aligned}
$$

The only contribution to the integrand above comes from terms quadratic in the fluctuations, so the gaussian integration over these oscillations will lead to a super-determinant. Because the localization manifold is purely bosonic, this super-determinant is simply equal to the ratio of two determinants, one associated with the fermionic fluctuations and the other with the bosonic fluctuations. These determinants can then be computed by explicit diagonalization, or by making use of powerful fixed-point formulas [39]. Of course, obtaining explicit expressions must be done in the context of a specific application.

We have presented the formula (5.9) including only the contributions from smooth field configurations. In addition, one must also allow for field configurations that are singular precisely at the fixed point in space-time of $\delta_{\xi}$, which in super-Yang-Mills theories, for instance, correspond to point-like instantons $[16,17]$.

\section{Application to exact quantum entropy of supersymmetric black holes}

In the previous sections we have been very general about the nature of the theory that we may wish to consider. In this closing section we therefore turn to a specific direction of interest that demonstrates how our construction of the equivariant algebra naturally lends itself to computing supersymmetric gravitational functional integrals in asymptotically AdS spaces, where the boundary conditions on the fields are dictated by the conformal boundary of the space [40]. The background can be chosen to be supersymmetric with an $\operatorname{AdS}_{n} \times S^{m}$ geometry and the commuting background ghosts are restricted to a particular supercharge on this background, and characterized by a generalized Killing spinor. This supercharge must be chosen such that its square leads to the compact background transformation $\delta_{\xi}$. This can be directly verified from the supersymmetry algebra of the supergravity under consideration and is required to be compact. The observables in our BRST cohomology in this case would be the holographic analogs of protected calculations in the boundary gauge theory ${ }^{9}$ which leads to an exciting possibility for an exact AdS/CFT correspondence.

To illustrate this idea in a concrete example, we revisit the analysis of $[20,21]$ of the quantum entropy of dyonic four-dimensional half-BPS black holes in $\mathcal{N}=2$ supergravity in the context of the formalism of this paper. Our construction of the equivariant algebra (4.6)

\footnotetext{
${ }^{9}$ Some classical aspects of a special class of such observables have been recently discussed in [46]; related ideas in a slightly different context of topological strings are discussed in [47].
} 
provides a proper framework for applying localization of the path integral for supergravity theories defined on spaces with an asymptotic boundary, as outlined in section 5. Hence it can in principle be applied to the path integral that defines the quantum entropy [19]. Further details of actual computations are reported in [45], but here our aim is to present an overview of this application in order to further clarify the formal discussions of the previous sections.

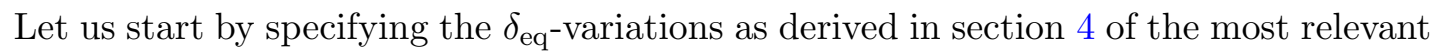
fermion fields in a purely bosonic field configuration in the context of the superconformal formulation of $\mathcal{N}=2$ supergravity $[49,50]$. These fermions belong to the Weyl and the vector supermultiplets. Here we will make use of the off-shell gauge algebra of Euclidean $\mathcal{N}=2$ superconformal gravity presented in [38]. The Weyl supermultiplet contains the gravitino fields whose $\delta_{\text {eq-variation in a bosonic field configuration equals }}$

$$
\delta_{\text {eq }} \psi_{\mu \pm}^{i}=2 \mathcal{D}_{\mu}\left(\varepsilon^{i}+c_{Q}^{i}\right)_{ \pm}+\frac{1}{16} \mathrm{i} T_{a b} \gamma^{a b} \gamma_{\mu}\left(\varepsilon^{i}+c_{Q}^{i}\right)_{\mp}-\mathrm{i} \gamma_{\mu}\left(\eta^{i}+c_{S}^{i}\right)_{\mp}
$$

where the subscript \pm on the fermions and ghosts denote chiral projections. The quantum ghosts associated with $Q$ - and $S$-supersymetry are $c_{Q}{ }^{i}$ and $c_{S}{ }^{i}$; at this point we leave the corresponding background ghosts $\varepsilon^{i}$ and $\eta^{i}$ unrestricted. Here and henceforth the indices $i, j, \ldots$ refer to the $\mathrm{SU}(2) \mathrm{R}$-symmetry. Note that we have suppressed the universal anticommuting parameter $\Lambda$ since, in a bosonic field configuration, there are no subtleties with relative signs of the various contributions. The other bosonic fields in these equations are the metric, the auxiliary tensor $T_{a b}$ as well as related gauge connections that are part of the off-shell Weyl multiplet. All these fields must be decomposed into background and quantum fields, as we have explained in previous sections. The off-shell Weyl multiplet also contains another fermion field, which we will ignore here because it only plays a minor role in what follows.

To describe the electric and magnetic charges of the black hole, the supergravity must include a number of vector supermultiplets labeled by $I=0 \ldots n_{\mathrm{v}}$. Their corresponding

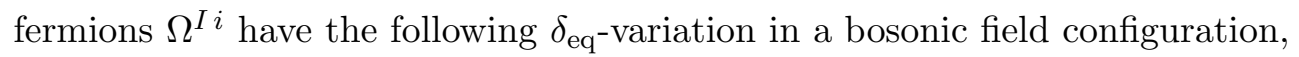

$$
\begin{aligned}
\delta_{\mathrm{eq}} \Omega_{ \pm}^{i I}= & 2 \mathrm{i} \not D X_{ \pm}{ }^{I}\left(\varepsilon^{i}+c_{Q}{ }^{i}\right)_{\mp}+\left[\frac{1}{2} \delta_{j}{ }^{i} \widehat{F}_{a b}{ }^{\mp I} \gamma^{a b}+\varepsilon_{k j} Y^{i k I}\right]\left(\varepsilon^{j}+c_{Q}{ }^{j}\right)_{ \pm} \\
& -2 X_{ \pm}{ }^{I}\left(\eta^{i}+c_{S}{ }^{i}\right)_{ \pm} .
\end{aligned}
$$

The right-hand side of the above equation contains the real scalar fields $X_{ \pm}^{I}$ and the auxiliary $\mathrm{SU}(2)$ triplets $Y^{i j I}$, whereas the gauge fields enter through the (anti-)selfdual projections of the modified field strength $\widehat{F}_{a b} I$. The covariant derivative on the scalars contains the various connections belonging to the Weyl multiplet.

Although the equations (6.1) and (6.2) represent the equivariant variations $\delta_{\text {eq }}$ of the quantum fermions, they reduce to the standard $Q$ - and $S$-supersymmetry transformations of the fermions prior to the background field split upon identifying the fields on the righthand side with the background fields and, at the same time, suppressing the quantum ghosts $c_{Q}{ }^{i}$ and $c_{S}{ }^{i}$, and keeping only the background ghosts $\varepsilon^{i}$ and $\eta^{i}$. Hence they can be used to exhibit the consequences of full supersymmetry for the near-horizon geometry. 
Since the background must be fully supersymmetric the truncated equations (6.1) and (6.2) must vanish for all values of the background ghosts $\varepsilon^{i}$ and $\eta^{i}$ (up to certain gauge choices). The result of this analysis is that the background geometry must be $\mathrm{AdS}_{2} \times S^{2}$ and that the full background is invariant under eight supersymmetries generated by particular linear combinations of $\varepsilon^{i}$ and $\eta^{i}$ that define eight independent Killing spinors associated with the fermionic isometries of the full background configuration. Such spinors are not normalizable in the asymptotic $\mathrm{AdS}_{2}$ space.

Obviously the above analysis leads precisely to the fully supersymmetric near-horizon geometry $\mathrm{AdS}_{2} \times S^{2}$ with fixed electric and magnetic fluxes [34, 48]. Here we should add that the background values of the gauge fields are constrained by the background values of the scalars $X_{ \pm}^{I}$. The gauge fields carry fixed electric and magnetic charges, corresponding to the microcanonical ensemble. The condition of fixed magnetic charges is implemented on the gauge field components along the $S^{2}$ in the asymptotic region. The condition of fixed electric charges is implemented in the classical theory by a Legendre transform with respect to the electric fields. In the quantum theory this requires the introduction of a Wilson line at the boundary of the near-horizon region, and we must compute the expectation value of this operator by integrating all fluctuations of all the supergravity fields around the above background [19].

Now we turn to the computation of the functional integral by following the localization procedure explained in section $5 .{ }^{10}$ For that purpose we have to determine the localization manifold, which follows from requiring the $\delta_{\text {eq }}$-variations of the quantum fermions, given by (6.1) and (6.2), to vanish. The background ghosts in these variations are then restricted to those associated to a particular supercharge. The latter can be identified with a supercharge generated by a Killing spinor of the background that asymptotes to a particular near-horizon Killing spinor. We note that the background and the quantum ghosts entering the $\delta_{\mathrm{eq}}$-variations provide us with a structure analogous to the one in [17], where localization was conducted using a combination of the BRST transformation and a specific background supersymmetry transformation. In our situation where gravity is dynamical, both these supercharges are naturally unified and encoded in a single $\delta_{\text {eq }}$ transformation.

In the presence of a single background ghost the $\delta_{\text {eq }}$-variations simplify. For the fully supersymmetric background under consideration, the associated Killing spinor can be chosen so that the square of the equivariant variations generates a background transformation $\delta_{\dot{\xi}}=L-J$, where $L$ and $J$ are compact abelian rotations of the $\mathrm{AdS}_{2}$ and $S^{2}$ factors in the background geometry, respectively. Thus, we can reduce the final problem to finding all geometries and bosonic matter field configurations that asymptote to the near-horizon fully supersymmetric $\mathrm{AdS}_{2} \times S^{2}$ background, and that admit a Killing spinor which asymptotes to the above near-horizon Killing spinor. This is precisely the problem addressed in [20] and solved in [41] for smooth field configurations. In order to complete the calculation of the functional integral (5.9), we then need to evaluate the physical action of the theory on these localizing configurations [20,21, 42], and we need to compute the one-loop fluctu-

\footnotetext{
${ }^{10}$ For $\mathrm{AdS}_{2}$ one has to take into account an additional subtlety coming from the fact that there are normalizable gauge transformations with corresponding non-normalizable gauge parameters [51].
} 
ation determinant [43-45]. Finally, as mentioned above, the smoothness assumption that we made in supergravity should be removed in string theory, wherein a class of orbifold configurations also contribute to the functional integral $[52,53]$.

It is clear that the quantum entropy problem for BPS black holes in asymptotically flat space is but one application of our ideas. The formalism constructed in this paper is quite general in that it can be defined around an arbitrary background that admits (super-)isometries. Our discussion gives a precise physical realization of the idea of equivariant cohomology, and of the corresponding equivariant localization using the background supersymmetry ghosts, in the variables of supergravity. We hope that the framework outlined in this paper will prove useful in a variety of other physical situations.

\section{Acknowledgments}

We thank Atish Dabholkar, Camillo Imbimbo, Rajesh Gupta, Imtak Jeon, Paul Richmond and Alberto Zaffaroni for interesting and useful discussions. B. de Wit and V. Reys thank King's College London for hospitality during the course of this work. The work of S. Murthy was supported by the ERC Consolidator Grant N. 681908, "Quantum black holes: A macroscopic window into the microstructure of gravity", and by the STFC grant $\mathrm{ST} / \mathrm{P} 000258 / 1$. V. Reys is supported in part by INFN and by the ERC Starting Grant 637844-HBQFTNCER.

Open Access. This article is distributed under the terms of the Creative Commons Attribution License (CC-BY 4.0), which permits any use, distribution and reproduction in any medium, provided the original author(s) and source are credited.

\section{References}

[1] R.P. Feynman, Quantum theory of gravitation, Acta Phys. Polon. 24 (1963) 697 [InSPIRE].

[2] B.S. DeWitt, Quantum theory of gravity. 2. The manifestly covariant theory, Phys. Rev. 162 (1967) 1195 [INSPIRE].

[3] L.D. Faddeev and V.N. Popov, Feynman diagrams for the Yang-Mills field, Phys. Lett. B 25 (1967) 29 [INSPIRE].

[4] C. Becchi, A. Rouet and R. Stora, Renormalization of the abelian Higgs-Kibble model, Commun. Math. Phys. 42 (1975) 127 [InSPIRE].

[5] I.V. Tyutin, Gauge invariance in field theory and statistical physics in operator formalism, arXiv:0812.0580 [INSPIRE].

[6] E.S. Fradkin and T.E. Fradkina, Quantization of relativistic systems with boson and fermion first and second class constraints, Phys. Lett. B 72 (1978) 343.

[7] B. de Wit and J.W. van Holten, Covariant quantization of gauge theories with open gauge algebra, Phys. Lett. 79B (1978) 389 [INSPIRE].

[8] I.A. Batalin and G.A. Vilkovisky, Gauge algebra and quantization, Phys. Lett. B 102 (1981) 27. 
[9] I.A. Batalin and G.A. Vilkovisky, Feynman rules for reducible gauge theories, Phys. Lett. B 120 (1983) 166.

[10] J. Gomis, J. Paris and S. Samuel, Antibracket, antifields and gauge theory quantization, Phys. Rept. 259 (1995) 1 [hep-th/9412228] [inSPIRE].

[11] E. Witten, Topological quantum field theory, Commun. Math. Phys. 117 (1988) 353 [INSPIRE].

[12] E. Witten, Topological $\sigma$-models, Commun. Math. Phys. 118 (1988) 411 [InSPIRE].

[13] J.J. Duistermaat and G.J. Heckman, On the variation in the cohomology of the symplectic form of the reduced phase space, Invent. Math. 69 (1982) 259.

[14] N. Berline and M. Vergne, Classes caractéristiques équivariantes. Formule de localisation en cohomologie équivariante, C. R. Acad. Sci. Paris Sér. I Math. 295 (1982) 539.

[15] M.F. Atiyah and R. Bott, The moment map and equivariant cohomology, Topology 23 (1984) 1 [INSPIRE].

[16] N.A. Nekrasov, Seiberg-Witten prepotential from instanton counting, Adv. Theor. Math. Phys. 7 (2003) 831 [hep-th/0206161] [INSPIRE].

[17] V. Pestun, Localization of gauge theory on a four-sphere and supersymmetric Wilson loops, Commun. Math. Phys. 313 (2012) 71 [arXiv:0712.2824] [INSPIRE].

[18] V. Pestun et al., Localization techniques in quantum field theories, J. Phys. A 50 (2017) 440301 [arXiv: 1608.02952] [INSPIRE].

[19] A. Sen, Quantum entropy function from $A d S_{2} / C F T_{1}$ correspondence, Int. J. Mod. Phys. A 24 (2009) 4225 [arXiv:0809.3304] [INSPIRE].

[20] A. Dabholkar, J. Gomes and S. Murthy, Quantum black holes, localization and the topological string, JHEP 06 (2011) 019 [arXiv: 1012.0265] [INSPIRE].

[21] A. Dabholkar, J. Gomes and S. Murthy, Localization \& exact holography, JHEP 04 (2013) 062 [arXiv:1111.1161] [INSPIRE].

[22] L. Baulieu and I.M. Singer, Topological Yang-Mills symmetry, Nucl. Phys. Proc. Suppl. B 5 (1988) 12.

[23] R.J. Szabo, Equivariant cohomology and localization of path integrals, Lect. Notes Phys. Monogr. 63 (2000) 1 [INSPIRE].

[24] P.K. Townsend, Covariant quantization of antisymmetric tensor gauge fields, Phys. Lett. 88B (1979) 97 [INSPIRE].

[25] W. Siegel, Hidden ghosts, Phys. Lett. 93B (1980) 170 [INSPIRE].

[26] B. de Wit and N. Papanicolaou, Spontaneous symmetry breaking of slavnov symmetry: a restriction on the gauge condition, Nucl. Phys. B 113 (1976) 261 [INSPIRE].

[27] K. Fujikawa et al., The regularized BRST coordinate invariant measure, Phys. Rev. D 37 (1988) 391 [INSPIRE].

[28] Z. Bern, E. Mottola and S.K. Blau, General covariance of the path integral for quantum gravity, Phys. Rev. D 43 (1991) 1212 [InSPIRE].

[29] G. 't Hooft and M.J.G. Veltman, Combinatorics of gauge fields, Nucl. Phys. B 50 (1972) 318 [INSPIRE]. 
[30] L. Baulieu, M. Bellon and V. Reys, Twisted $N=1, d=4$ supergravity and its symmetries, Nucl. Phys. B 867 (2013) 330 [arXiv:1207.4399] [inSPIRE].

[31] J. Bae, C. Imbimbo, S.-J. Rey and D. Rosa, New supersymmetric localizations from topological gravity, JHEP 03 (2016) 169 [arXiv: 1510.00006] [INSPIRE].

[32] K. Costello and S. Li, Twisted supergravity and its quantization, arXiv:1606.00365 [INSPIRE].

[33] C. Imbimbo and D. Rosa, The topological structure of supergravity: an application to supersymmetric localization, JHEP 05 (2018) 112 [arXiv:1801.04940] [INSPIRE].

[34] G. Lopes Cardoso, B. de Wit, J. Kappeli and T. Mohaupt, Stationary BPS solutions in $N=2$ supergravity with $R^{2}$ interactions, JHEP 12 (2000) 019 [hep-th/0009234] [INSPIRE].

[35] B. de Wit, S. Katmadas and M. van Zalk, New supersymmetric higher-derivative couplings: full $N=2$ superspace does not count!, JHEP 01 (2011) 007 [arXiv: 1010.2150] [INSPIRE].

[36] D. Butter, B. de Wit, S.M. Kuzenko and I. Lodato, New higher-derivative invariants in $N=2$ supergravity and the Gauss-Bonnet term, JHEP 12 (2013) 062 [arXiv:1307.6546] [INSPIRE].

[37] A.S. Schwarz and O. Zaboronsky, Supersymmetry and localization, Commun. Math. Phys. 183 (1997) 463 [hep-th/9511112] [INSPIRE].

[38] B. de Wit and V. Reys, Euclidean supergravity, JHEP 12 (2017) 011 [arXiv:1706.04973] [INSPIRE].

[39] M.F. Atiyah, Elliptic operators and compact groups, Lecture Notes in Mathematics volume 401, Springer, Germany (1974).

[40] E. Witten, Anti-de Sitter space and holography, Adv. Theor. Math. Phys. 2 (1998) 253 [hep-th/9802150] [INSPIRE].

[41] R.K. Gupta and S. Murthy, All solutions of the localization equations for $N=2$ quantum black hole entropy, JHEP 02 (2013) 141 [arXiv: 1208.6221] [INSPIRE].

[42] S. Murthy and V. Reys, Quantum black hole entropy and the holomorphic prepotential of $N=2$ supergravity, JHEP 10 (2013) 099 [arXiv: 1306.3796] [INSPIRE].

[43] S. Murthy and V. Reys, Functional determinants, index theorems and exact quantum black hole entropy, JHEP 12 (2015) 028 [arXiv: 1504.01400] [INSPIRE].

[44] R.K. Gupta, Y. Ito and I. Jeon, Supersymmetric Localization for BPS Black Hole Entropy: 1-loop Partition Function from Vector Multiplets, JHEP 11 (2015) 197 [arXiv:1504.01700] [INSPIRE].

[45] I. Jeon and S. Murthy, Twisting and localization in supergravity: equivariant cohomology of BPS black holes, arXiv: 1806.04479 [INSPIRE].

[46] P. Benetti Genolini, P. Richmond and J. Sparks, Topological AdS/CFT, JHEP 12 (2017) 039 [arXiv:1707.08575] [INSPIRE].

[47] T.D. Brennan, F. Carta and C. Vafa, The string landscape, the swampland and the missing corner, arXiv:1711.00864 [INSPIRE].

[48] G. Lopes Cardoso, B. de Wit and T. Mohaupt, Corrections to macroscopic supersymmetric black hole entropy, Phys. Lett. B 451 (1999) 309 [hep-th/9812082] [InSPIRE]. 
[49] B. de Wit and A. Van Proeyen, Potentials and symmetries of general gauged $N=2$ supergravity: Yang-Mills models, Nucl. Phys. B 245 (1984) 89 [INSPIRE].

[50] B. de Wit, P.G. Lauwers and A. Van Proeyen, Lagrangians of $N=2$ supergravity - Matter systems, Nucl. Phys. B 255 (1985) 569 [INSPIRE].

[51] N. Banerjee et al., Supersymmetry, localization and quantum entropy function, JHEP 02 (2010) 091 [arXiv: 0905.2686] [INSPIRE].

[52] N. Banerjee, D.P. Jatkar and A. Sen, Asymptotic expansion of the $N=4$ dyon degeneracy, JHEP 05 (2009) 121 [arXiv: 0810.3472] [INSPIRE].

[53] S. Murthy and B. Pioline, A Farey tale for $N=4$ dyons, JHEP 09 (2009) 022 [arXiv: 0904 .4253] [INSPIRE]. 